\title{
Thermo-Structural Analysis of Carbon Fibre-Ni Based Super Alloy Composite Employed in Gas Turbines
}

\author{
Ankit Dhoka \\ Department of Aerospace Engineering, SRM University, Chennai, Tamil Nadu, India \\ Email address: \\ Ankitdhoka987@gmail.com \\ To cite this article: \\ Ankit Dhoka. Thermo-Structural Analysis of Carbon Fibre-Ni Based Super Alloy Composite Employed in Gas Turbines. American Journal \\ of Mechanical and Materials Engineering. Vol. 4, No. 3, 2020, pp. 60-65. doi: 10.11648/j.ajmme.20200403.14
}

Received: August 2, 2020; Accepted: August 27, 2020; Published: September 7, 2020

\begin{abstract}
A computational analysis was carried out on two different materials of turbine blades, namely Inconel MA754 and Nimonic 80A, in order to determine their structural and thermal properties at elevated temperatures. Long carbon fibers of uniform length were used and deposited at varying thicknesses ranging from $1 \mathrm{~mm}$ to $4 \mathrm{~mm}$, on the top surface of turbine blades and then analyzed for its performance. It is seen that the carbon fibers (IM10) embedded in the super alloys drastically improve the load bearing parameters of the configurations being analyzed. The improvement in structural load carrying ability is a result of higher Young's modulus primarily. Subsequent analysis with higher volume fraction of the fibers indicated saturation of performance at about $70 \%$ volume fraction for $4 \mathrm{~mm}$ fibers and significant improvement beyond it for the $1 \mathrm{~mm}$ fibers. With improvement in the load bearing characteristics the blade with fibers embedded into a tube like structure at 3 sections were configured and A thermal analysis of the same underscores the effectiveness of the $4 \mathrm{~mm}$ fibers in undergoing much reduced principal strains than other configurations. This is seen to be a result of insulation of the top surface from increase in temperature, which significantly reduces the thermal expansion, especially at the free end. This is in contrast to other configurations, where the low volume fraction of fibers resulted in high principal strain.
\end{abstract}

Keywords: Carbon Fibers, FEM, Turbine Blades, Thermal Analysis, High Temperature Materials

\section{Introduction}

The crux of modern day aircraft propulsive performance is related to ideas of clean combustion, higher operating pressures, and temperatures for turbines. Among the forementioned ideas, the role of higher operating turbine temperatures has probably the most impact on the success of the other two ideas. This is evident from the compressor, that derives its work from turbine and thus is intrinsically linked. Cleaner combustion is constrained by the turbine inlet temperature to a certain degree, and thus the whole geometry of combustion chamber liners and dilution holes are associated with the choice of materials for turbine blades. The two widely approached ideas on improving turbine operating temperature are -1 . Turbine cooling methods and 2 . Development of materials that can withstand high temperatures, albeit with the aid of cooling.

Cooling methods can be active and passive, with the latter involving coating of heat resistant materials or coatings, while active methods employ controlled cooling. Widely used active cooling methods employ internal and external cooling, with the cooling fluid running across the span of the tubine blade. [1-2] The external cooling is more effective, owing to non-interference from the thermal diffusive nature of the turbine blades. The popular film cooling employs several holes tapped on the surface of the turbine blade, which create a thin surface film flowing over the turbine blade. This technique results in overall drop of temperature by around $200^{\circ} \mathrm{C}$. The holes are preferred along the leading edge, owing to highest heat transfer flux being present there.

The advent of superalloys, either Nickel, Cobalt or Iron based has led to development of hard, strong and highly temperature resistant materials. These have been the purpose of active research [3-5]. The ability of the superalloys to increase their yield strength, with higher temperatures owing to the locking of the disloacations result in effective hardening. However, after a certain time, turbine blades made of superalloys can undergo degradation, due to a combination of stress and temperature effects resulting in both dislocation and diffusion creep. Although superalloys are extremely strong at 
high temperatures owing to the dislocation lock, inhomogenities in the process of manufacture can render creep effects dominant as a result of different grain sizes. Methods have been devised to counter this, by employing solutes in grain boundaries, notably carbides to inhibit boundary sliding. A recent method adopted by [6] incorporates application of magnetic field to ensure homogeneous composition of the Nickel based super-alloy through two phases.

As a further development, passive cooling methods were employed along-with superalloys. Notable among them is the thermal barrier coating applied over turbine blades. Typically, they span with-in range of 100 micrometer to 2 millimeter and offer oxidation and thermal fatigue resistance to the blade material However as illustrated in [7-8], these thermal barrier coatings develop typical compressive stress and creep stress, that results in changes in the material properties with time, and thus to eventual failure. In order to address the above concern, materials with high creep resistance and better thermal deformation performance were chosen in the form of superalloys and ceramic composites. The process involves the embedding of carbide fibres in carbide matrix, notably Silicon Carbide ( $\mathrm{SiC}$ ) [9-11]. Owing to the refractory nature of such materials, the strength and thermal characteristics have been seen to be vastly improved to temperatures as high as $1450^{\circ} \mathrm{C}$.

A possible replacement of carbide fibers in turbine blades can be carbon fibres, which have high tensile strength, low thermal expansion coefficient as well as high temperature tolerance. These fibers have already been used in aircraft structural aspects ranging from B-787 to space shuttles. The superior corrosion resistance has also enabled carbon fibers to replace Aluminium from structural components. The performance of carbon composites and the role of advanced manufacturing and addition of other allotropes like carbon nanotubes in structural applications has also been detailed in [12-13].

The present paper is an extension of the fore-mentioned concepts, by application of carbon fibers embedded in super alloys and determine the thermal and structural stability of the same. The work deals with the computational analysis of the same across two superalloys, both Nickel based and is aimed towards optimizing the volume fraction and length of carbon fibers to obtain the best performance in the listed constraint space.

\section{Computational Methodology}

The materials that have been taken into consideration for analysis are the Nickel based superalloys-, Inconel MA754 and Nimonic $80 \mathrm{~A}$. The super alloys are chosen based on their popularity and application in gas turbine enviromnents as well as rocket engines. The super alloys form the base, or the embedded structure, through which the carbon fibers can be placed, by means of suitable adhesive in reality. In the present work, the fibers and the embedded material are modelled in CATIA $15^{\circledR}$, whereby the two components of the turbine blade are rigid w.r.t each other.

The steady state thermo-structural computational analysis have been performed on Ansys ${ }^{\circledR} \mathrm{v}$ 16.0, with the airfoil cross-section being chosen as NACA 4412. To allow for future experimental studies on lab scale, the length of the blade is chosen as $117 \mathrm{~mm}$ and the chord being equal to $32 \mathrm{~mm}$. The angle of attack is chosen as 5 degrees, which in contextual terms is the relative angle of attack. Since, the analysis is being performed from the blade frame of reference, the actual forces need to be corrected for pseudo forces. However, the present work is not intended to cover those aspects and only the steady analysis of the forces on the blade, considered from the blade frame of reference is performed. A total of three blades have been designed, with one blade having no carbon fiber on the blade, one blade with $1 \mathrm{~mm}$ of thickness on top of the blade, and the last blade with $4 \mathrm{~mm}$ of thickness on top surface of the blade. The carbon fibers correspond to the IM10 variant. The fibers act as analogs of stringers, in actual wings. The overall length of the chord has been kept the same while applying layer of carbon fiber by resizing the initial length of the chord. The length of the carbon fibers spanning from one chord length to a third of the chord length followed similar operation. The side and top view of the model is shown in Figure 1. The volume fraction of the fibers to the total volume fraction is considered as 50, 60 and 70\%. The six different material configurations are listed in Table 1.

Table 1. Material configurations.

\begin{tabular}{lllll}
\hline Material 1 & Material 2 & Material 3 & Material 4 & Material 5 \\
\hline Inconel MA754 & Nimonic $80 \mathrm{~A}$ & Inconel 1mm fibers & Nimonic 1mm fibers & Inconel 4mm fibers \\
\hline
\end{tabular}

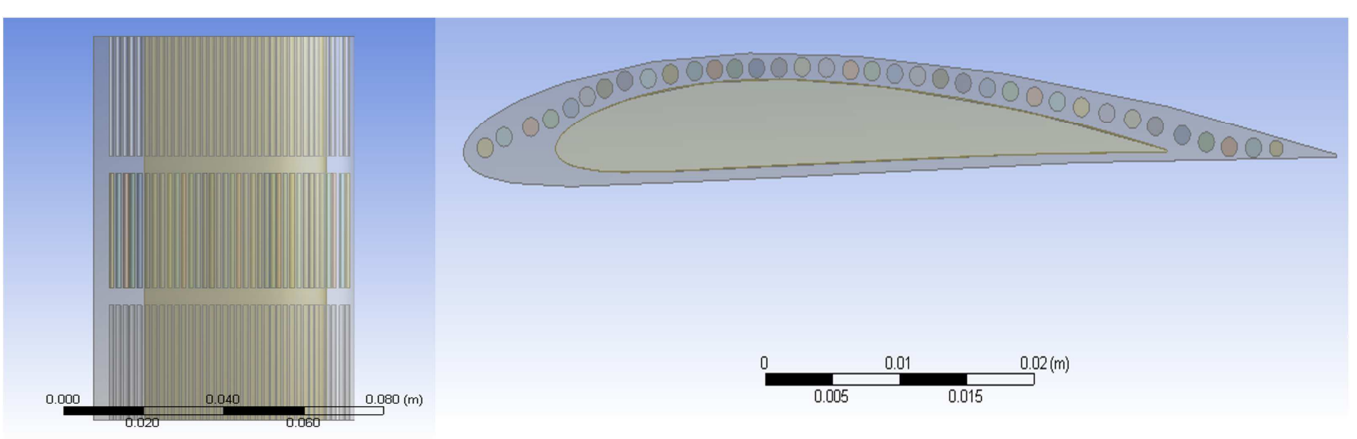

Figure 1. Top view (left) and side view (right), with segmented embedded Carbon fibers and on top surface. 
The material properties of all these materials have been fed into the material library of the Ansys ${ }^{\circledR}$ workbench and are tabulated in Table 1. A fine structured mesh, arrived after grid independence study and computational cost optimization of element size of $1 \mathrm{~mm}$ has been chosen in the present work. The corresponding number of nodes is 368605 and the number of elements are 2080758.

To provide the input force, the blade momentum theory, was applied considering the axial and radial induction factor to be zero. The velocities were scaled, from the usual velocities found in actual turbines $(50 \mathrm{~m} / \mathrm{s})$ to ensure constant Reynolds number. The results show that the force acting on the blade along the transverse direction to the blade is $440 \mathrm{~N}$ for a single blade $25 \mathrm{~N}$ along the stream-wise direction. The blades are treated as cantilever type, with the hub being a fixed support, and the forces assumed to be uniformly distributed over the span of the blade. Although this assumption is weird for a constant angle of attack profile, in reality, uniform forces can be provided by suitable blade twist. This procedure has been carried out for all the three blades.

\section{Results and Discussion}

The results are broadly distinguished into structural and thermal deformation, with the former performed fist, to check the effect of different configurations on the parameters related to the stress and strain. The results with pure superalloys and their composites with Carbon fibers, when the fibers span the entire length of the blade is tabulated in Table 2.

It is seen that Nimonic has a better overall load bearing ability, compared to Inconel, thus it is obvious that if one considers from a computational perspective, whereby fabrication issues are neglected, any material improvement over Nimonic will be deemed worthy. It is apparent then, that addition of Carbon fibers, of any thickness seems to increase the load bearing ability of the super-alloys. The effect of larger carbon fibers, which eventually lead to larger volume fraction of the surface is more drastic, with an order of magnitude lower stress and strains, associated with both principal stresses (normal stress) as well as shear stress. The reasoning of lower strains, both principal and shear is pretty obvious. From the material properties of the constituent materials, the Young's modulus of the IM10 carbon fibers is significantly higher than either Nimonic $80 \mathrm{~A}$ or Inconel MA754, with the latter having least among all.

Table 2. Load parameters for the different material configurations.

\begin{tabular}{lllllll}
\hline Parameters & Material 1 & Material 2 & Material 3 & Material 4 & Material 5 & Material 6 \\
\hline Total Deformation & 0.00209 & 0.00205 & 0.0013 & 0.00125 & 0.000348 & 0.000344 \\
Principal stress (GPa) & 0.321 & 0.307 & 0.360 & 0.340 & 0.0839 & 0.0830 \\
Shear stress (GPa) & 0.0142 & 0.0112 & 0.0101 & 0.0082 & 0.00186 & 0.00184 \\
Principal strain & 0.0017 & 0.0016 & 0.0013 & 0.00129 & 0.00045 & 0.00041 \\
\hline
\end{tabular}

The marginal difference in the Young's modulus of Nimonic and Inconel variants result in near comparable performance. Hence, for a given stress that will be described later, the strains will be lower, either principal or shear for the carbon fiber embedded super alloys. Another key aspect of carbon fibers is its low Poisson's ratio, which is nearly half of that of Inconel, that results in higher directional/ isotropic shear modulus as given by the shear modulus- Young's modulus relation. The higher shear modulus, derived from Poisson's ratio as well as significantly higher Young's modulus, ensure low structural strain, for a given stress configuration. One should note that, owing to low Poisson's ratio, the amount of normal strain, for a given state stress that can be lowered in super alloys is not possible in context of Carbon fibers embedded super alloys.

In order to understand the stress variation, the stress variation in the cross-section at the free end for the case of material 6 is shown in Figure 2. The relation of these stressstrain fields to other material configurations will be evident from the figures as well.
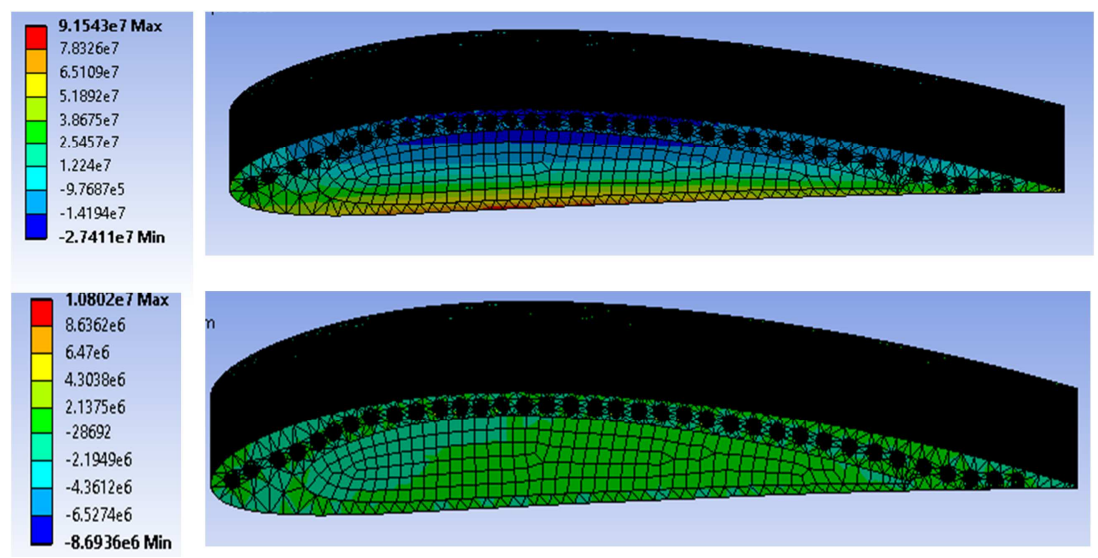

Figure 2. (Top) Principal stress and (bottom) shear stress across the cross-section for material 6. 
Since the stress is primarily in the transverse direction, and from the principal stress value plots as seen from Figure 2 (top), it is clear that the primary component of the principal stress is the bending stress, with the values shifting from tension on the bottom side (positive) to compression (negative), with the neutral axis closely following the chord line. The peak principal stress, is towards the top surface, or where the curvature peaks, owing to more distance from neutral axis as well as higher curvatures, which lead to higher stress concentrations. In-fact the positions of leading edge and trailing edge, incidentally coincide with the neutral axis, that ensures that the stress values in the vicinity is low. This presents an important observation, especially for trailing edge, where the curvatures are highest, that it is susceptible to failure, followed by the airfoil surface corresponding to camber and the leading edge. Design of highly skewed airfoils, especially design of airfoils with surfaces like flaps and slots, increases the tendency for failure by stress concentration.

Compared to the principal stress, whereby the major component is from the bending (normal) stress, the shear stress presents a slightly complicated figure. The shear stress is seen to be negative (producing anti-clock-wise moment), in the vicinity of the leading edge, with progression towards a positive shear stress from nearly a quarter chord point till the trailing edge. Considering the fact, that the only imposed loads are the forces along the stream-wise and transverse direction, the shear stress originates from the loads themselves, and as a result of the bending moments. which in the two dimensional plane constitutes a set of two moments. The resultant shear arises as measure to counter or to provide equilibrium to the variation of the bending stress across the span of the blade. In terms of any general cross -section expressed in the Cartesian coordiate, we can write the general expression for shear stress as,

$$
\begin{aligned}
& \frac{\partial \tau_{y x}}{\partial x}-\frac{\partial \sigma_{x}}{\partial x}=0 \\
& \frac{\partial \tau_{x y}}{\partial y}-\frac{\partial \sigma_{y}}{\partial y}=0
\end{aligned}
$$

Thus, the variation of the shear stress, $\tau_{y x}$ and $\tau_{x y}$, along $\mathrm{x}$ and y directions (stream-wise and transverse) at any point ( $x$, $y$ ) within the control area is governed by the above set of Partial differential equations. The coaxial bending stresses are $\sigma_{x}$ and $\sigma_{y}$. The variation of the normal stresses is governed by the Euler-Bernoulli equation. Considering that the beam is not slender, the beam can be modeled as a Timoshenko beam. However, since the load distribution is constant, the resulting equation for both the formulations for the current problem remains the same. One knows, that the bending stress magnitude depends inversely on the Young's modulus for a given load, and thus, as a result, the normal stress distribution remains similar for all the materials listed formerly, with the values scaling only due to differences in Young's modulus and differences in strain coming as a result of the same as well as Poisson's ratio. By analyzing equations (1-2), we can conclude that the variation in the shear stress is mainly a result of the switch in the bending stresses along the respective coordinate directions (primarily across neutral axes). The variation of shear stress and bending moment across the span of the structure is a direct consequence of the moment that is generated at any section by the stream-wise and transverse loads.

Once the idea of higher volume fraction of carbon fibers, especially in the top surface proving to be effective in withstanding higher loads, and thus in reducing principal strains, we explore the effect of increasing the volume fraction of carbon fibers on top compared to the overall volume. The results are presented in Table 3 . The results are presented for Nimonic $80 \mathrm{~A}$ owing to its superior characteristics.

Table 3. Load parameters for listed material variants.

\begin{tabular}{lllll}
\hline Parameters & Material 4 (60\%) & Material 4 (70\%) & Material 5 (60\%) & Material 5 (70\%) \\
\hline Total Deformation & 0.0020639 & 0.0020369 & 0.00030816 & 0.00030813 \\
Principal stress (GPa) & 0.337 & 0.324 & 0.0797 & 0.0770 \\
Shear stress (GPa) & 0.0150 & 0.00943 & 0.00175 & 0.0017 \\
Principal strain & 0.0015 & 0.00084 & 0.00037 & 0.00037 \\
\hline
\end{tabular}

As expected, one can observe that the higher volume fraction of carbon fibers significantly increases the load bearing capacity across all the parameters of interest. The rest of the paper, however is devoted to analysis with the base value of $50 \%$.

In order to study, the effects of raised temperature on the structure, the blade is subjected to two different high temperatures, at $1350 \mathrm{~K}$ and $1870 \mathrm{~K}$. The increase in temperature, results in generation of both stress and strain, as a result of free expansion and the fixed surface, and is related to the resultant coefficient of thermal expansion, which is very low for IM10 carbon fibers. The result is tabulated in Table 4. For higher temperature, the trends are the same, except for enhanced magnitudes of all the listed load parameters. It is very interesting to note that, there is one order of magnitude rise in the principal stress, followed by a two order of magnitude increase in the shear stress. This is accompanied by a near constant total deformation across temperatures, which can be attributed to the strain inhibition provided by the reaction support provided by the fixed ends. 
Table 4. Load parameters at $1350 \mathrm{~K}$.

\begin{tabular}{lllllll}
\hline Parameters & Material 1 & Material 2 & Material 3 & Material 4 & Material 5 & Material 6 \\
\hline Total Deformation & 0.0025623 & 0.0025374 & 0.0011373 & 0.001135 & 0.0003347 \\
Principal stress (GPa) & 2.97 & 2.70 & 5.71 & 5.11 & 0.000344 \\
Shear stress (GPa) & 1.62 & 1.42 & 1.82 & 1.80 & 0.0840 \\
Principal strain & 0.04473 & 0.034681 & 0.0306 & 0.0277 & 0.00248 \\
\hline
\end{tabular}

The transmission of this reaction stress across the span of the blade can be the only reason for the near invariance of total deformation. Even if the total deformation is low, one can observe significant increase in the principal strains, which can be wholly explained on its direct relation to the square root of the shear strain, and thus shows one order of magnitude higher strain. However, it is interesting to note that even at such high temperatures, IM10 Carbon fibers of higher tube diameter perform exceptionally well, with shear stress and principal strain being nearly same as that of the purely structural load configuration.

In order to understand, the dynamics of thermal and structural loads in a coupled fashion, let us observe at the cross-section as shown in Figure 3. Contrasted to the approach of looking at the stresses acting on the cross-section in Figure 2, here we resort to observation of the principal strain, owing to its fundamental relationship to change in volume, experienced by a heated material. However, in order to compare across two different material configurations we inspect, the behavior of material 4 and material 6 .

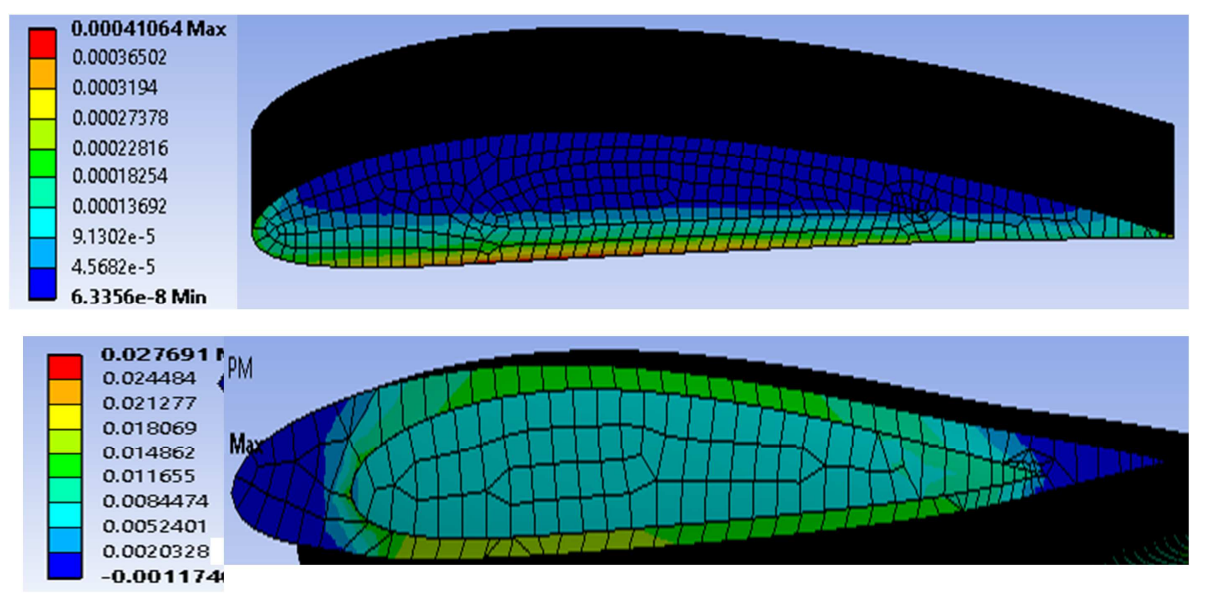

Figure 3. (Top) Principal strain for material 6 and (bottom) material 4 at $1350 \mathrm{~K}$.

From Figure 3, it is interesting to observe the stark contrast in the regions corresponding to maximum principal strain are different across the two materials. For material 4, the bulk of principal strain occurs at the top and bottom surfaces, which are actually associated with the regions of maximum strain for a purely structural load experiencing member. However, the distinction is in the fact, that the edges of the surface possess highest temperature and hence the highest strain, more so being a free end. The minimum occurs at the leading and the trailing edge, which are in-fact regions corresponding to the neutral axis. The interesting aspect worth pointing out here is that the leading and trailing edge, present high values of heat flux, owing to their curvature, however that doesn't reflect as increased principal strains. This could be due to directional expansion of the elemental volumes on the periphery of the leading edge more than the interior (due to higher temperature), that results in overall compression of most of the region and results in a negative principal strain. The same argument can be extended to the trailing edge for material 4.

For the case of material 6 , the trends seems to be the same at the bottom side, however, on the top side, where a majority of the volume fraction is taken over by higher diameter carbon fibers, the principal strain becomes a minimum. This is owing to the low thermal conductivity of the fibers, that ensures that the temperature does not increase, nor is conducted to the interior. The overall result is low principal strains. However, the lower surface, has the usual traits of higher strains at the periphery, followed by lower principal strain in the interior.

The above results indicate at the effectiveness of carbon fibers, in effectively reducing the thermo-structural loads, at significantly higher temperatures. The issues related to carbon fiber is its ability to adhere to the super alloys, especially at high temperatures and its ability to crack under compression, which however is not significant as seen from the results.

\section{Conclusion}

A computational analysis of a three dimensional turbine blade from the rotor frame of reference was analyzed. The analyses performed involve application of purely structural loads, by filling the top surface of the airfoil with carbon fibers (IM10) of different diameters. It was seen that these fibers embedded in the Nickel based super alloys Nimonic 80 A and Inconel MA 754 performed better than the base super alloys across principal load parameters. The primary reason 
for the superior performance was found to be the high Young's modulus and associated low Poisson's ratio, that explained lower strains noted for a given load. In the context of stress distribution, the primary stress contributing to the principal stresses were the bending stress, which when governed by the Euler Bernoulli theory, would yield lower values for the Carbon fibers embedded super alloys. The performance of the materials was seen to improve with higher volume stacking of the Carbon fibers with exceptional performance noted for the higher diameter $4 \mathrm{~mm}$ configuration.

Subsequently, the analysis was performed for higher temperatures, in the presence of the same load. The analysis indicated that, the $4 \mathrm{~mm}$ fibers performed exceptionally well, comparable to pure structural loading configurations. The distinction between other configurations and the $4 \mathrm{~mm}$ configuration was seen to be primarily the insulation of the top surface from increase in temperature. For the other configurations, higher principal strains were noted in the top regions of the airfoil, which are suppressed in the case of the fiber being embedded and occupying a large volume fraction. The extension of this work, in an experimental sense would ensure development and limitations of Carbon fiber embedded in super alloys as a suitable material for the use in gas turbines, whereby higher operating temperatures will result in higher efficiency of the plant.

\section{References}

[1] Eckert, E. R. G., 1984. Analysis of film cooling and fullcoverage film cooling of gas turbine blades. Journal of Engineering for Gas Turbines and Power, 106 (1), pp. 206213.

[2] Ebenhoch, G. and Speer, T. M., 1996. Simulation of cooling systems in gas turbines. Journal of turbomachinery, 118 (2), pp. 301-306.

[3] Choudhury, I. A., and M. A. El-Baradie. "Machinability of nickel-base super alloys: a general review." Journal of Materials Processing Technology 77. 1-3 (1998): 278-284.
[4] Caron, P. and Khan, T., 1999. Evolution of Ni-based superalloys for single crystal gas turbine blade applications. Aerospace Science and Technology, 3 (8), pp. 513-523.

[5] Henderson, M. B., Arrell, D., Larsson, R., Heobel, M. and Marchant, G., 2004. Nickel based superalloy welding practices for industrial gas turbine applications. Science and Technology of Welding and Joining, 9 (1), pp. 13-21.

[6] Ren, W., Niu, C., Ding, B., Zhong, Y., Yu, J., Ren, Z., Liu, W., Ren, L. and Liaw, P. K., 2018. Improvement in creep life of a nickel-based single-crystal superalloy via composition homogeneity on the multiscales by magnetic-field-assisted directional solidification. Scientific reports, 8 (1), p. 1452.

[7] Evans, A. G., Mumm, D. R., Hutchinson, J. W., Meier, G. H. and Pettit, F. S., 2001. Mechanisms controlling the durability of thermal barrier coatings. Progress in materials science, 46 (5), pp. 505-553.

[8] Rösler, J., Bäker, M. and Volgmann, M., 2001. Stress state and failure mechanisms of thermal barrier coatings: role of creep in thermally grown oxide. Acta materialia, 49 (18), pp. 36593670 .

[9] Mehan, R. L. and Bolon, R. B., 1979. Interaction between silicon carbide and a nickel-based superalloy at elevated temperatures. Journal of materials science, 14 (10), pp. 24712481.

[10] Cao, X. Q., Vassen, R. and Stoever, D., 2004. Ceramic materials for thermal barrier coatings. Journal of the European Ceramic Society, 24 (1), pp. 1-10.

[11] Belmonte, M., 2006. Advanced ceramic materials for high temperature applications. Advanced engineering materials, 8 (8), pp. 693-703.

[12] De Volder, M. F., Tawfick, S. H., Baughman, R. H. and Hart, A. J., 2013. Carbon nanotubes: present and future commercial applications. Science, 339 (6119), pp. 535-539.

[13] Gohardani, O., Elola, M. C. and Elizetxea, C., 2014. Potential and prospective implementation of carbon nanotubes on next generation aircraft and space vehicles: a review of current and expected applications in aerospace sciences. Progress in Aerospace Sciences, 70, pp. 42-68. 